\title{
Distinct epigenetic features of tumor- reactive CD8+ T cells in colorectal cancer patients revealed by genome-wide DNA methylation analysis
}

Rui Yang ${ }^{1+}$, Sijin Cheng ${ }^{1+}$, Nan Luo ${ }^{2,3+}$, Ranran Gao ${ }^{1}$, Kezhuo Yư ${ }^{4}$, Boxi Kang ${ }^{1}$, Li Wang $^{1}$, Qiming Zhang ${ }^{1}$, Qiao Fang ${ }^{4}$, Lei Zhang ${ }^{4}$, Chen $\mathrm{Li}^{5}$, Aibin $\mathrm{He}^{5}$, Xueda $\mathrm{Hu}^{1}$, Jirun Peng ${ }^{2,3^{*}}$, Xianwen Ren ${ }^{1^{*}}$ and Zemin Zhang ${ }^{1,4^{*}}$ (D)

\begin{abstract}
Background: Tumor-reactive CD8+ tumor-infiltrating lymphocytes (TILS) represent a subtype of T cells that can recognize and destroy tumor specifically. Understanding the regulatory mechanism of tumor-reactive CD8+ T cells has important therapeutic implications. Yet the DNA methylation status of this T cell subtype has not been elucidated.

Results: In this study, we segregate tumor-reactive and bystander CD8+ TILs, as well as naïve and effector memory CD8+ T cell subtypes as controls from colorectal cancer patients, to compare their transcriptome and methylome characteristics. Transcriptome profiling confirms previous conclusions that tumor-reactive TILs have an exhausted tissue-resident memory signature. Whole-genome methylation profiling identifies a distinct methylome pattern of tumor-reactive CD8+ T cells, with tumor-reactive markers CD39 and CD103 being specifically demethylated. In addition, dynamic changes are observed during the transition of naïve T cells into tumor-reactive CD8+ T cells. Transcription factor binding motif enrichment analysis identifies several immune-related transcription factors, including three exhaustion-related genes (NR4A1, BATF, and EGR2) and VDR, which potentially play an important regulatory role in tumor-reactive CD8+ T cells.

Conclusion: Our study supports the involvement of DNA methylation in shaping tumor-reactive and bystander CD8+ TILs, and provides a valuable resource for the development of novel DNA methylation markers and future therapeutics.
\end{abstract}

Keywords: Colorectal cancer, Tumor-reactive T cells, Bystanders, Transcriptome, Methylome, Transcription factor, T cell exhaustion

\section{Background}

Colorectal cancer (CRC) is one of the most common cancers globally. CRC incidence has traditionally been the highest in affluent western countries, but it is now increasing rapidly in other countries with economic

\footnotetext{
*Correspondence: pengjr@medmail.com.cn; renxwise@pku.edu.cn; zemin@pku.edu.cn

${ }^{\dagger}$ Rui Yang, Sijin Cheng and Nan Luo contributed equally to this work. ${ }^{2}$ Department of Surgery, Beijing Shijitan Hospital, Capital Medical University, Beijing, China

'BIOPIC, Beijing Advanced Innovation Center for Genomics, and School of Life Sciences, Peking University, Beijing, China

Full list of author information is available at the end of the article
}

development. CRC treatment usually involves surgical removal of the tumor followed by adjuvant chemotherapy. In recent years, various kinds of immunotherapies, such as checkpoint blockade immunotherapy, have been used to enhance the antitumor potential. However, the responses to these treatments vary among patients. Recent literatures supported the notion that not all tumorinfiltrating lymphocytes (TILs) are tumor reactive [1-4]. Rather, bystander cells exist, which recognize a wide range of epitopes unrelated to cancer [1-4]. For tumor immunotherapy, it is valuable to target those cells of which the $\mathrm{T}$ cell receptor (TCR) repertoire is

(c) The Author(s). 2019 Open Access This article is distributed under the terms of the Creative Commons Attribution 4.0 International License (http://creativecommons.org/licenses/by/4.0/), which permits unrestricted use, distribution, and reproduction in any medium, provided you give appropriate credit to the original author(s) and the source, provide a link to the Creative Commons license, and indicate if changes were made. The Creative Commons Public Domain Dedication waiver (http://creativecommons.org/publicdomain/zero/1.0/) applies to the data made available in this article, unless otherwise stated. 
intrinsically tumor reactive. Co-expression of CD39 (ENTPD1) and CD103 (ITGAE) identifies such a unique $\mathrm{T}$ cell population $[1,3]$. These cells have a tissueresident memory (RM) signature with high expression of exhaustion markers, such as PDCD1 and HAVCR2 (also known as Tim-3). Interestingly, these TILs also exhibited low expression of CCR7, CD127, and CD28, indicative of an effector memory (EM) phenotype $[3,5]$. Understanding the molecular basis of memory CD8 $+\mathrm{T}$ cells is key to developing effective therapies against cancers. Further investigation is needed to better distinguish the molecular natures of $\mathrm{T}_{\mathrm{EM}}$ and this tumor-reactive $\mathrm{T}$ cell subtype.

Gene expression patterns, a key determinant for a cellular feature, are believed to be controlled by epigenetic changes [6]. Decoding the epigenome specific to tumorreactive $T$ cells is a pivotal step toward understanding the activation and expansion of this $\mathrm{T}$ cell population in cancer. However, how they are regulated epigenetically has not been addressed thus far. DNA methylation, a covalent modification of the DNA molecule, is a stable and heritable form of epigenetic modifications which participates in establishing and maintaining chromatin structures and regulates gene transcription [7]. In general, DNA methylation is critical for establishing stable genesilencing programs, by affecting the interactions of DNA with chromatin proteins and transcription factors $[8,9]$. Many studies have highlighted the importance of DNA methylation in regulating complex gene expression programs underlying immune responses [10-12]. It is thus important to define how the identities of tumor-reactive $\mathrm{CD} 8+\mathrm{T}$ cells and bystanders are shaped at methylation level, including particular genes and networks.

In this study, we sorted tumor-reactive and bystander CD8+ TILs from treatment-naïve primary CRC patients based on the expression of CD39 and CD103, and naïve and $\mathrm{T}_{\mathrm{EM}} \mathrm{CD} 8+\mathrm{T}$ cells from peripheral blood based on the expression of CD45RO, CD45RA, and CCR7. Adapted smart-seq2 and whole-genome bisulfite sequencing (bisulfite-seq) were performed to characterize the transcriptomic features, DNA methylome programming, methylation dynamics, and key transcription factors (TFs) in each T cell subtype. Our study can help understand the underlying mechanisms leading to the specific expression patterns of tumor-reactive CD8+ $\mathrm{T}$ cells, thereby facilitating the development of new therapeutic strategies targeting these cells.

\section{Results}

Transcriptomic characteristics of five CD8+ T cell subtypes

Within CD8+ TILs, CD103+CD39+ T cells have been recently demonstrated to be tumor-reactive, while CD103-CD39- T cells and CD103+CD39- T cells are bystanders [1, 3]. To further characterize the transcriptional profiles of these cell populations, we isolated naïve, $\mathrm{T}_{\mathrm{EM}}, \mathrm{CD} 103+\mathrm{CD} 39+, \mathrm{CD} 103+\mathrm{CD} 39-$, and $\mathrm{CD} 103$ -CD39- $T$ cell subtypes from eight CRC patients for gene expression profiling using adapted Smart-seq II (Fig. 1a-c; Additional file 1: Figure S1A, B; the "Methods" section). As shown in the heat map displaying differentially expressed genes (DEGs) among five $\mathrm{CD} 8+\mathrm{T}$ cell subtypes, the naïve subtype exhibited high expression of known naïve markers $L E F 1$ and $S E L L$ (also known as CD62L) (Fig. 1d; Additional file 1: Figure $\mathrm{S} 1 \mathrm{C}) . \mathrm{T}_{\mathrm{EM}}$ subtype showed enhanced expression of classically defined $\mathrm{T}_{\mathrm{EM}}$ molecules, such as TBX21 [13] and CX3CR1 [14] (Fig. 1d). Notably, CD103+CD39+ TILs displayed hallmarks of an "exhausted" phenotype, with high expression of CTLA4, HAVCR2, and LAYN (Fig. 1d; Additional file 1: Figure S1C, D). Recent literatures reported that the thymocyte selection-associated high mobility group box (TOX) protein is required for the development and maintenance of exhausted $\mathrm{T}$ cell populations in chronic infection [15-18]. Removal of its DNA binding domain reduced the expression of PD-1 and resulted in a more polyfunctional $\mathrm{T}$ cell phenotype [16]. Here, we observed that TOX expression is also upregulated (Fig. 1d; Additional file 1: Figure S2A). Intriguingly, our previous single-cell RNA-sequencing (scRNA-seq) data identified the specific expression of TOX in exhausted CD8+ TILs [19-21] (Additional file 1: Figure S2B-D). These data together supported the important role of TOX in intratumoral T cell exhaustion.

Gene set variation analysis (GSVA) showed that CD103+CD39+ subtype was enriched in biological processes associated with immunomodulation, such as "regulation of interferon gamma biosynthesis" and "negative regulation of IL10 production" [22, 23] (Fig. 1e). Furthermore, we analyzed effector function of these CD8 $T$ cell subtypes by the expression of granzyme $\mathrm{A} / \mathrm{B} / \mathrm{H}$, cytotoxic granules PRF1, interferon (IFN)$\gamma$, and tumor necrosis factor (TNF). Interestingly, we found that exhausted CD103+CD39+ subtype still had relatively high expression of these cytotoxic proteins (Additional file 1: Figure S1C). Together with the GSVA results, it indicates that $\mathrm{CD} 103+\mathrm{CD} 39+$ subtype may not have lost their antitumor potential. Two-dimensional principal component analysis (PCA) revealed that naïve and $\mathrm{T}_{\mathrm{EM}}$ subtypes were clearly grouped as distinct populations, whereas three CD8+ TIL subtypes appeared tightly clustered, indicative of a very similar transcriptional profile among these subtypes (Fig. 1f).

To gain a deeper understanding of tumor-reactive CD8+ T cells, we compared them with their counterpart, CD103-CD39- cells. CD103+CD39+ T cells highly expressed a set of 435 genes, including $\mathrm{T}$ cell exhaustion markers CTLA4 and HAVCR2 (Fig. 1g), but they 


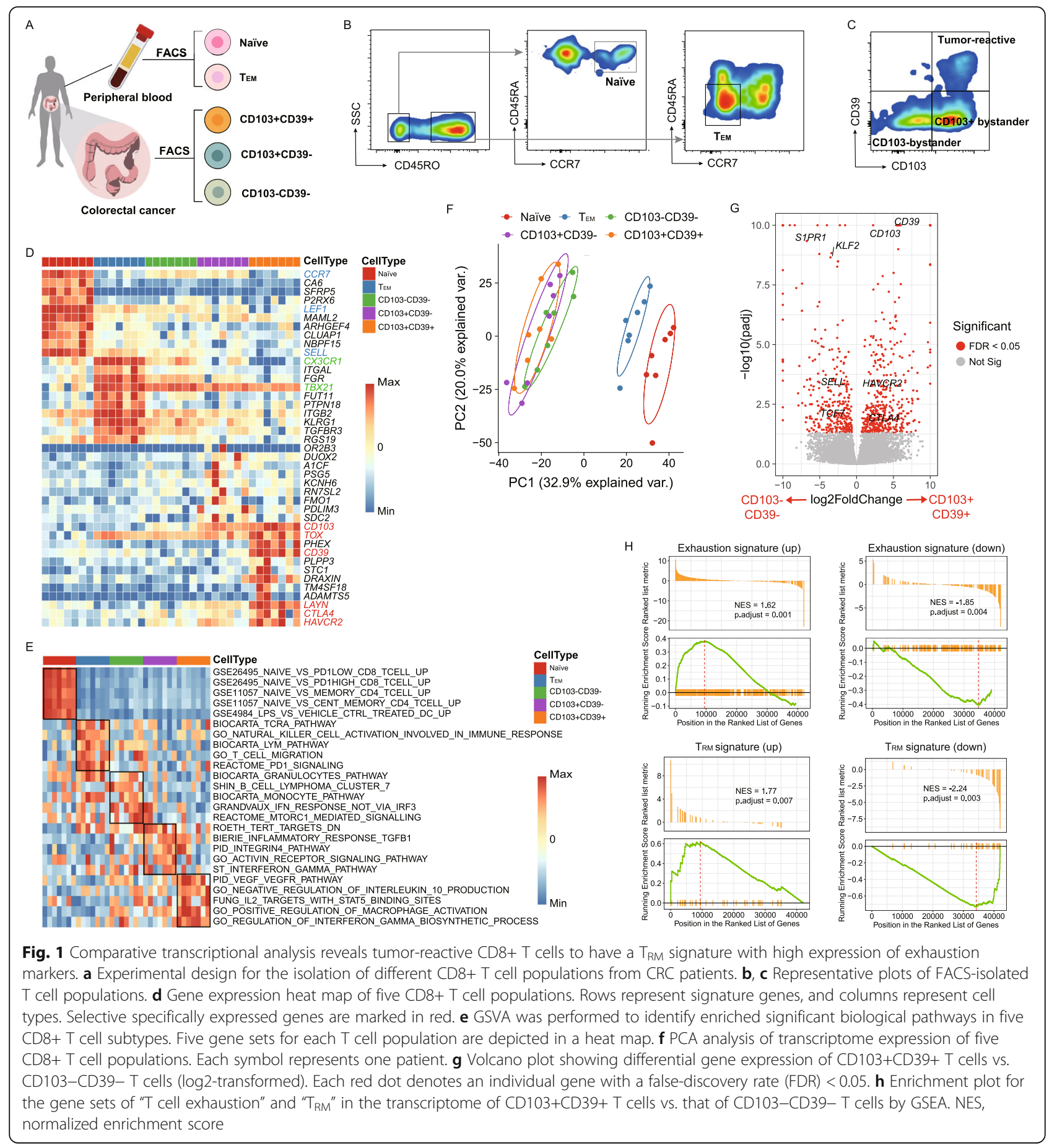

exhibited lower expression of genes involved in $\mathrm{T}$ cell recirculation, such as KLF2, SELL, and S1PR1 (Fig. 1g). Gene set enrichment analysis (GSEA) also revealed the presence of a molecular signature associated with $\mathrm{T}$ cell exhaustion and $\mathrm{T}_{\mathrm{RM}}$ signatures (Fig. $1 \mathrm{~h}$ ). Then, we compared the transcriptome of CD103+CD39+ TILs with that of the $\mathrm{T}_{\mathrm{EM}}$ subtype. Interestingly, CD103+CD39+ TILs also exhibited lower expression of CCR7, CD127, and $C D 28$, as did $\mathrm{T}_{\mathrm{EM}}$ subtype, consistent with previous findings (Additional file 1: Figure S1C) [3]. In addition, using a list of $\mathrm{T}_{\mathrm{EM}}$ signature genes [5], we found most of these genes to exhibit low expression in naïve $\mathrm{T}$ cell subtype and high expression in other subtypes (Additional file 1: Figure S1E), further confirming the $\mathrm{T}_{\mathrm{EM}}$ features in CD103+CD39+ T cells. Collectively, we again validated that tumor-reactive $\mathrm{CD} 8+\mathrm{T}$ cells are in an 
exhausted state, characteristic of both $T_{R M}$ and $T_{E M}$ features.

\section{Global DNA methylation profiling across five CD8+ T cell subtypes}

The phenotypic and functional changes that occur during CD8+ $\mathrm{T}$ cell differentiation are accompanied by genome-wide changes in DNA methylation programming. To comprehensively assess such methylation changes, we performed a genome-wide measurement of DNA methylation using bisulfite-seq (see the "Methods" section). For all samples, a median of $\sim 26$ million CpGs (45.1\%) was covered (Additional file 1: Table S1). PCA analysis of the CpG methylation level of 5 kilobase $(\mathrm{kb})$ genomic tiles among these $\mathrm{T}$ cell subtypes revealed that naïve $\mathrm{CD} 8+\mathrm{T}$ cells were clearly grouped as a distinct population, whereas the rest were clustered (Fig. 2a; Additional file 1: Figure S3A). The $\mathrm{T}_{\mathrm{EM}}$ subtype showed a similar methylation pattern to both bystander populations (Fig. 2a). Among the three TIL subtypes, CD103+CD39- T cells seem to possess a methylation signature that is intermediate between the CD103-CD39 - and CD103+CD39+ T cells CD8+ T cells (Fig. 2a). Statistical analysis revealed that naïve subtype has the highest methylation level, which is line with its quiescent state (Additional file 1: Figure S3B).

To characterize the methylome further, we calculated hypomethylated regions (HypoMRs) for each $\mathrm{T}$ cell population in a "one vs. rest" fashion. We found a total of 23,230 HypoMRs in all CD8+ T subtypes (Fig. 2b). Of note, signature genes $L E F 1, T C F 7$, and SELL in naive subtype and TBX21 and CX3CR1 in $\mathrm{T}_{\mathrm{EM}}$ subtype were affected by specific HypoMRs, which corresponded to their enhanced expression (Fig. 2c; Additional file 1: Figure S4A, B). ISG15, a ubiquitin-like interferonstimulated gene, was affected by a HypoMR in the CD103-CD39- subtype (Fig. 2c; Additional file 1: Figure S5). Its role as a central player in the host antiviral response might make it key to the immune functions of bystanders [24]. Notably, CD103+CD39+ T cells exhibited specific HypoMRs that affected the markers for tumor reactivity, CD39 and CD103 (Fig. 2c, e; Additional file 1: Figure S5). In addition, they also acquired an exhaustion-associated methylation program, with HypoMRs that affected the exhaustion markers $P D C D 1$, HAVCR2, and LAYN (Fig. 2c; Additional file 1: Figure S5). Our methylation data suggested that the cell features observed in different CD8+ T cell subtypes may be shaped by altered DNA methylation profiles.

\section{The methylation dynamics of immune-related genes}

To understand the dynamic changes of methylation during the development of tumor-reactive CD8+ T cells, we analyzed promoter methylation levels of three immune signature gene sets for naïve, cytotoxic, and exhausted T cells (Fig. 3a; Additional file 1: Figure S6). We found that most signature genes for naïve $\mathrm{T}$ cells were demethylated in naïve subtypes, and displayed a higher level of methylation in other later $\mathrm{T}$ cell subtypes (Fig. 3a; Additional file 1: Figure S6). Of note, TCF7 showed the most drastic changes (Fig. 3a; Additional file 1: Figure S6). In contrast, signature genes for cytotoxic $\mathrm{T}$ cells, including PRF1, IFNG, GZMB, CCL3, CCL4, NKG7, and CST7, were highly methylated in the naïve subtype and then became demethylated during naïve to $\mathrm{T}_{\mathrm{EM}}$ differentiation (Fig. 3a; Additional file 1: Figure S6), indicating that a hypomethylation programming was acquired following the activation of naïve $\mathrm{T}$ cells. The hypomethylation statuses of cytotoxic signature genes were maintained within both bystander and tumor-reactive CD8+ T cells (Fig. 3a; Additional file 1: Figure S6). Finally, for exhausted signature genes, two inhibitory receptors PDCD1 and CTLA4 were found to be specifically demethylated within tumor-reactive CD8+ T cells (Fig. 3a; Additional file 1: Figure S6). Another inhibitory receptor $L A G 3$ was initially methylated in naïve cells, and became demethylated in later stages of $\mathrm{T}$ cell subtypes (Fig. 3a; Additional file 1: Figure S6). LAYN, which was reported as a novel exhausted marker in our previous study [20], underwent striking methylation during naïve to $T_{E M}$ differentiation, maintained this methylation status in both bystander subtypes, and acquired demethylation in tumor-reactive $\mathrm{T}$ cells (Fig. 3a; Additional file 1: Figure S6).

Furthermore, to link DNA methylation states to gene expression, we performed correlation analyses of promoter methylation level and gene expression level. In most cases, the gene expression was found to be negatively correlated with their differential methylation status (Fig. 3b-d). For example, the expression level of PDCD1 showed significant negative correlation with the methylation level of its promoter region $(p=5.7 \mathrm{e}-12)$. The similar methylation levels of S1PR1 and SELL across different subtypes suggested that DNA methylation has a minor influence on their expression, and other factors may be responsible for their differential expression. In summary, the DNA methylation levels of immunerelated genes were in dynamic changes from naïve $\mathrm{T}$ cells to tumor-reactive CD8+ $\mathrm{T}$ cells, resulting in divergent expression programs in each $\mathrm{CD} 8+\mathrm{T}$ cell subtype.

\section{Key transcription factors for each CD8+ T cell subtype}

Transcription factors (TFs) have a broad effect on cell state. To gain insight into the key regulators in each CD8+ T cell subtype, we performed TF binding motif enrichment analysis in HypoMRs. Those enriched TFs might have decisive roles in shaping the molecular features for each $\mathrm{CD} 8+\mathrm{T}$ cell subtype. We found an 


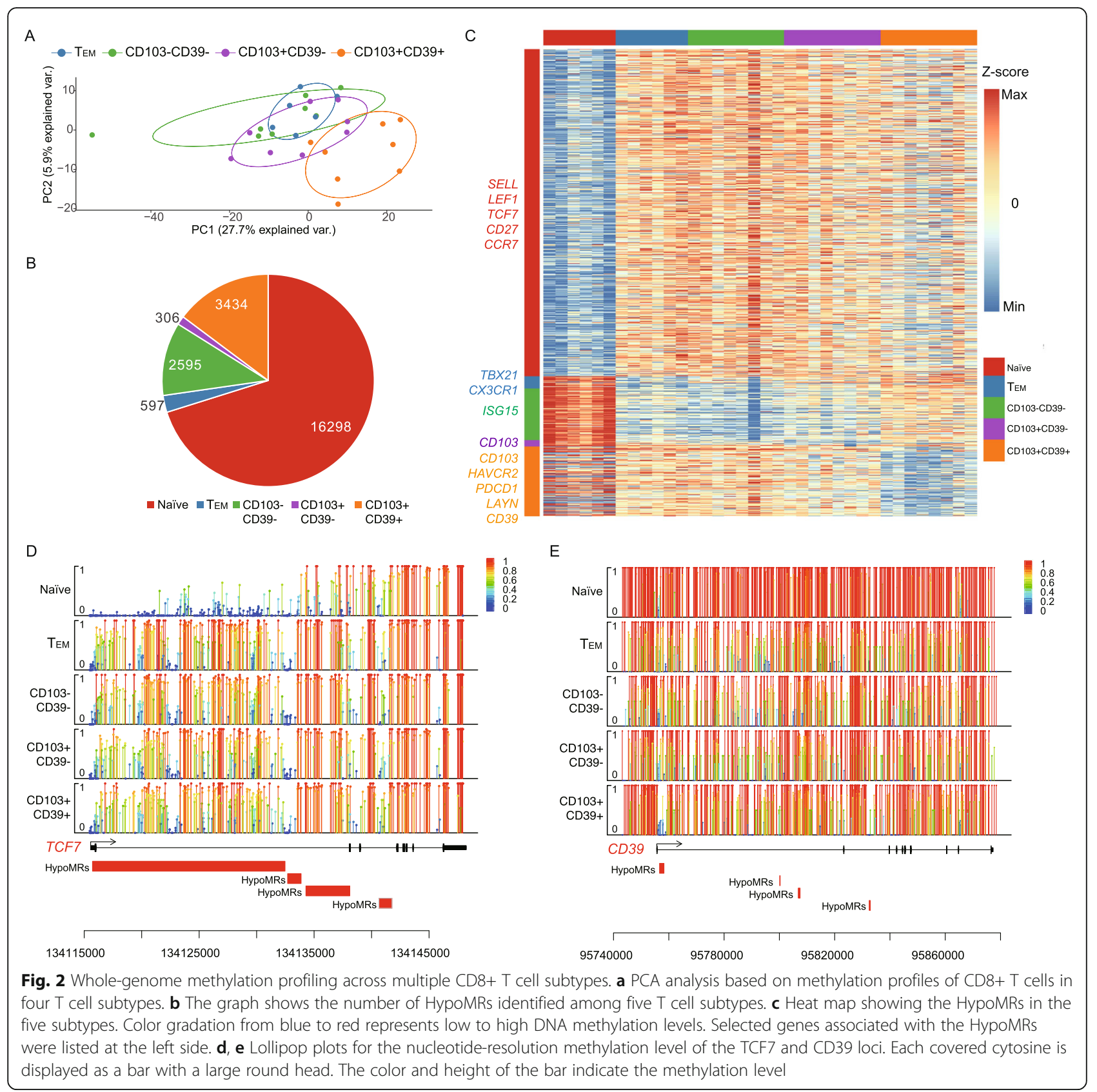

overrepresentation of binding sites of LEF1 and TCF7 in naïve subtype (Fig. 4a), consistent with their key regulatory functions in naïve $\mathrm{T}$ cells $[25,26]$. In both $\mathrm{T}_{\mathrm{EM}}$ and CD103-CD39- T cells, the binding motifs of two T-box TFs TBX21 and EOMES, which have been recognized as the master regulators of $\mathrm{CD} 8+\mathrm{T}$ cell differentiation and function [13, 27], were found to be enriched within HypoMRs (Fig. 4a). Additionally, BATF, a transcription factor in the $A P-1$ family, was found to be enriched within both CD103+CD39- and CD103+CD39+ TILs. $B A T F$ has been reported to initiate CD8+ $\mathrm{T}$ cell effector differentiation $[28,29]$. This is consistent with the notion that increased expression of BATF in exhausted CD8+ T cells suppresses their effector function $[29,30]$.

Particularly, this analysis yielded 85 TF binding motifs that were significantly enriched in $\mathrm{CD} 103+\mathrm{CD} 39+\mathrm{T}$ cells. In addition to $B A T F$, significantly overrepresented motifs included RUNX1, NR4A1 (also known as NUR77), vitamin D receptor (VDR), and EGR2 (Fig. 4a, b), suggesting that these cells are under the control of a complex network of transcription factors. Of these five TFs, BATF, NR4A1, and EGR2 have been reported to be associated with $\mathrm{T}$ cell exhaustion. BATF and NR4A1 could regulate PD-1 to inhibit $\mathrm{T}$ cell function [30, 31]; 


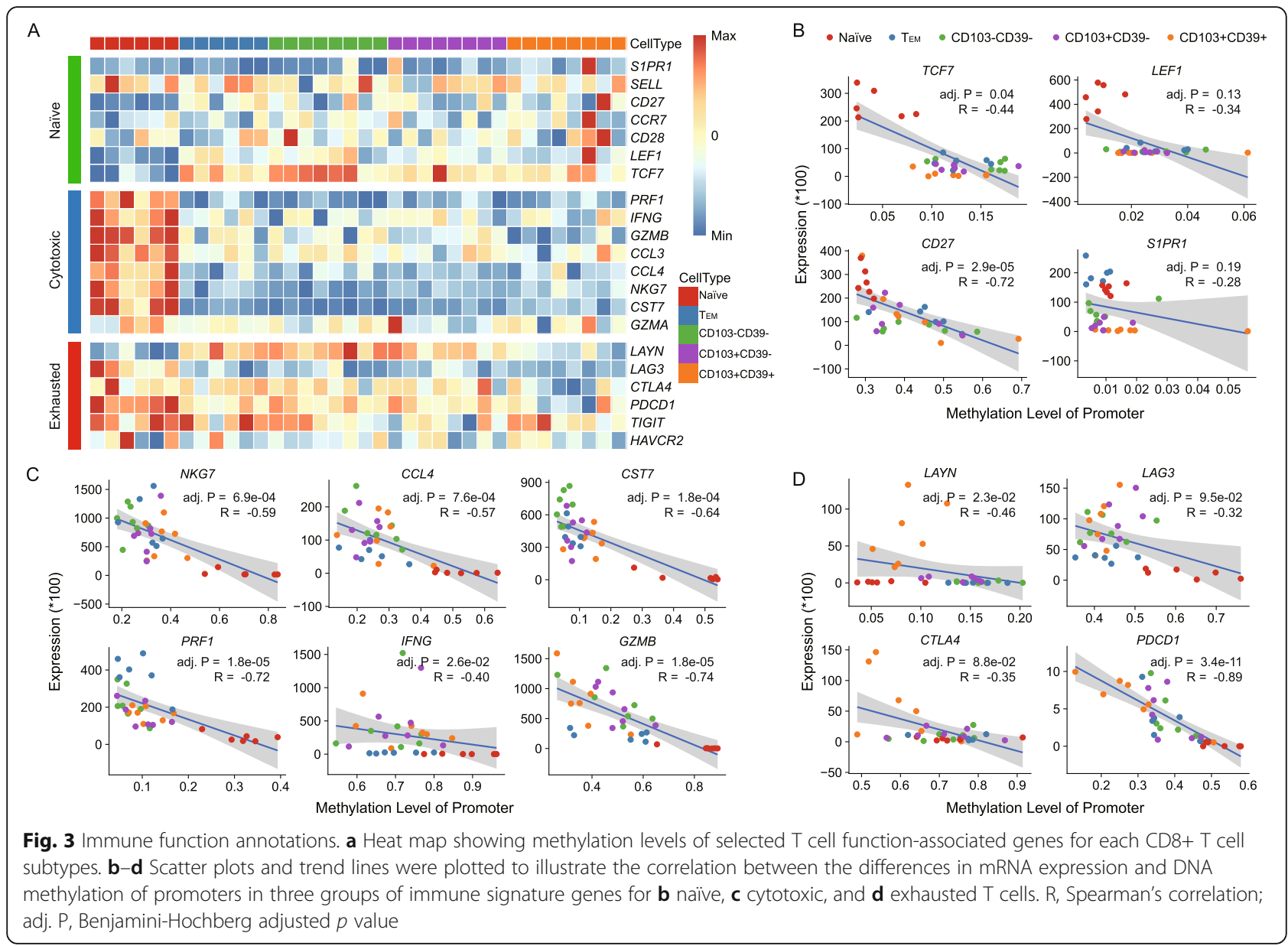

EGR2 targets $L A G 3$ and $4-1 B B$ regulate $T$ cell dysfunction within tumors [32]. The other two TFs, RUNX1 and $V D R$, have both been reported to be associated with $\mathrm{T}$ cell development $[33,34]$. Interestingly, RUNX1 mediates site-specific DNA demethylation at binding site in hematopoietic cells [35], which might explain the demethylated status here. Based on our RNA-Seq data, $B A T F, N R 4 A 1, V D R$, and EGR2 all showed high expression in CD103+CD39+ subtype (Fig. 4c). Notably, the expression level of VDR showed progressive increase during $\mathrm{T}$ cell differentiation from naïve to tumorreactive subtype. In contrast, we observed comparable expression of RUNX1 among five subtypes (Additional file 1: Figure S7A).

Next, to understand the transcriptional regulatory networks, we used TF binding motifs to predict targets regulated by TFs in tumor-reactive CD8+ T cells (see the "Methods" section). Of note, exhaustion markers $L A Y N$, HAVCR2, and PDCD1 and tissue-resident marker CD103 are predicted as the targets of these TFs (Fig. 4d-f; Additional file 1: Figure S7B, C). For example, $P D C D 1$ was predicted to be regulated by $V D R$, which has not been previously linked to $\mathrm{T}$ cell exhaustion. The regulatory role of $V D R$ in $P C D C 1$ regulation and $\mathrm{T}$ cell exhaustion thus deserves further interrogation. Other predicted targets include $\mathrm{T}$ cell trafficking molecule PDLIM4 and TNFRSF18 (GITR) which is involved in regulating $\mathrm{T}$ cell programmed cell death (Fig. 4d, f; Additional file 1: Figure S7C) [36]. Collectively, these data support that tumor-reactive CD8+ T cells were regulated by a complex network of transcription factors.

\section{Transcription factors in regulating $\mathrm{T}$ cell exhaustion}

Several predicted TFs in tumor-reactive cells have been reported to be associated with $\mathrm{T}$ cell exhaustion, such as NR4A1 and BATF [30,31]. In our study, a novel transcription factor $V D R$ was identified to be upregulated within tumor-reactive cells. Since PDCD1 upregulation is a hallmark of CD8+ T cell exhaustion, we here show an example of $P D C D 1$ regulated by three TFs to further discuss their relationship with $\mathrm{T}$ cell exhaustion. The recruitment of NR4A1 by PDCD 1 was supported by ChIPseq in a recent study [31]. Overall, a significant positive correlation existed between $P D C D 1$ expression and expression of NR4A1, BATF, and VDR in our own data (Fig. 5a). Particularly, when taking the promoter 


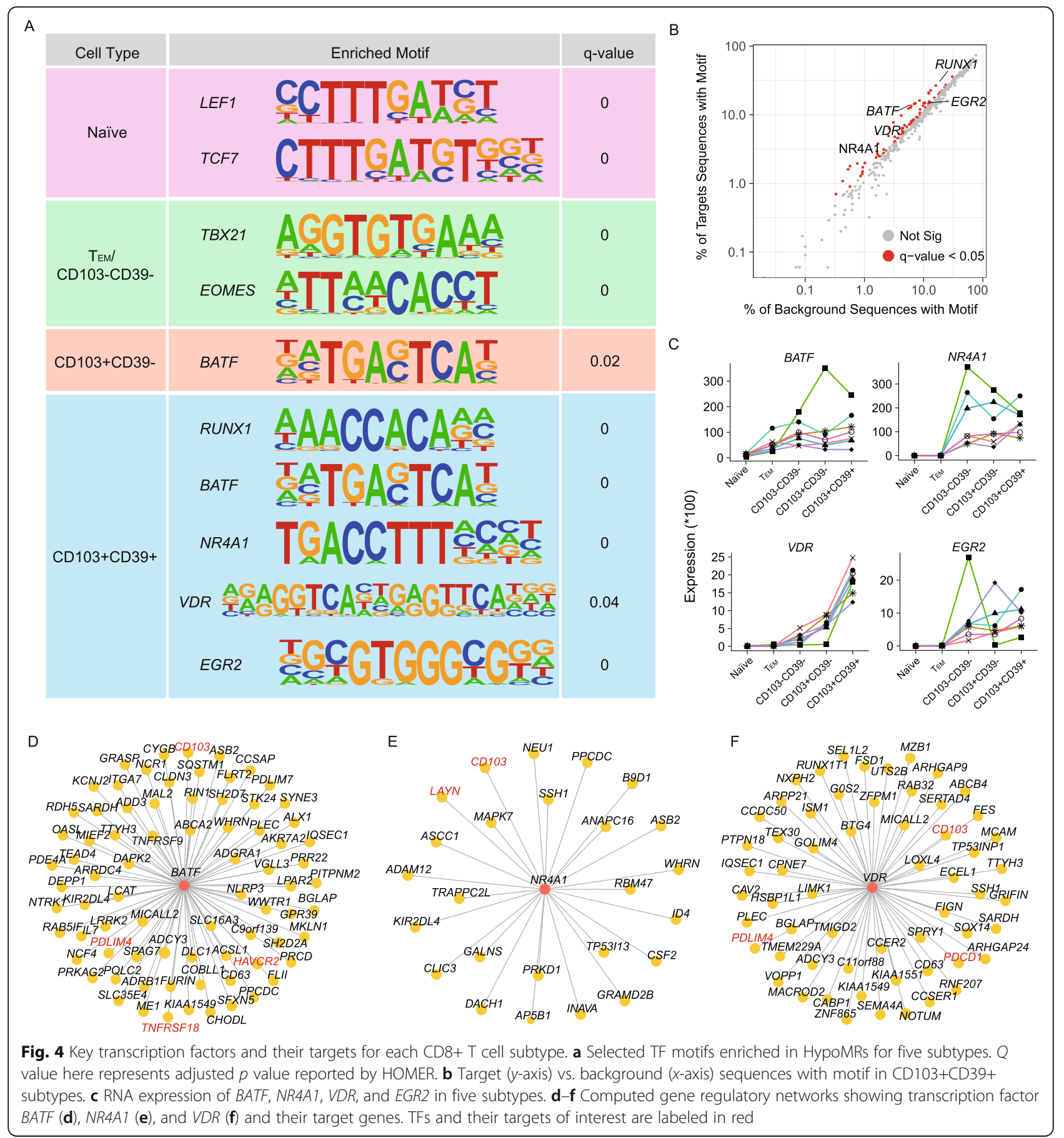

methylation level of $P D C D 1$ into account, several low expressing PDCD1 cells with high NR4A1 and VDR expression were found to have high DNA methylation levels of $P D C D 1$ promoters (Fig. 5a), suggesting the dominant role of DNA methylation in regulation $P D C D 1$ expression in these samples.

We used our previous scRNA-seq data of CD8+ T cells from CRC patients to further investigate the correlation of PDCD1 and three TFs [19]. In silico FACS analysis was used to predict the percentages of doublepositive cells for PDCD1 and TF in the corresponding TF-positive cells among three TIL subtypes, respectively. Of note, the highest proportions were observed in CD103+CD39+ subtype for all three TFs (Fig. 5b), which further supported the positive regulation of $P D C D 1$ by three TFs. Overall, our study indicated the transcription regulation of $P D C D 1$ in $C D 103+C D 39+$ subtype by both TF expression and DNA methylation state of TF binding 


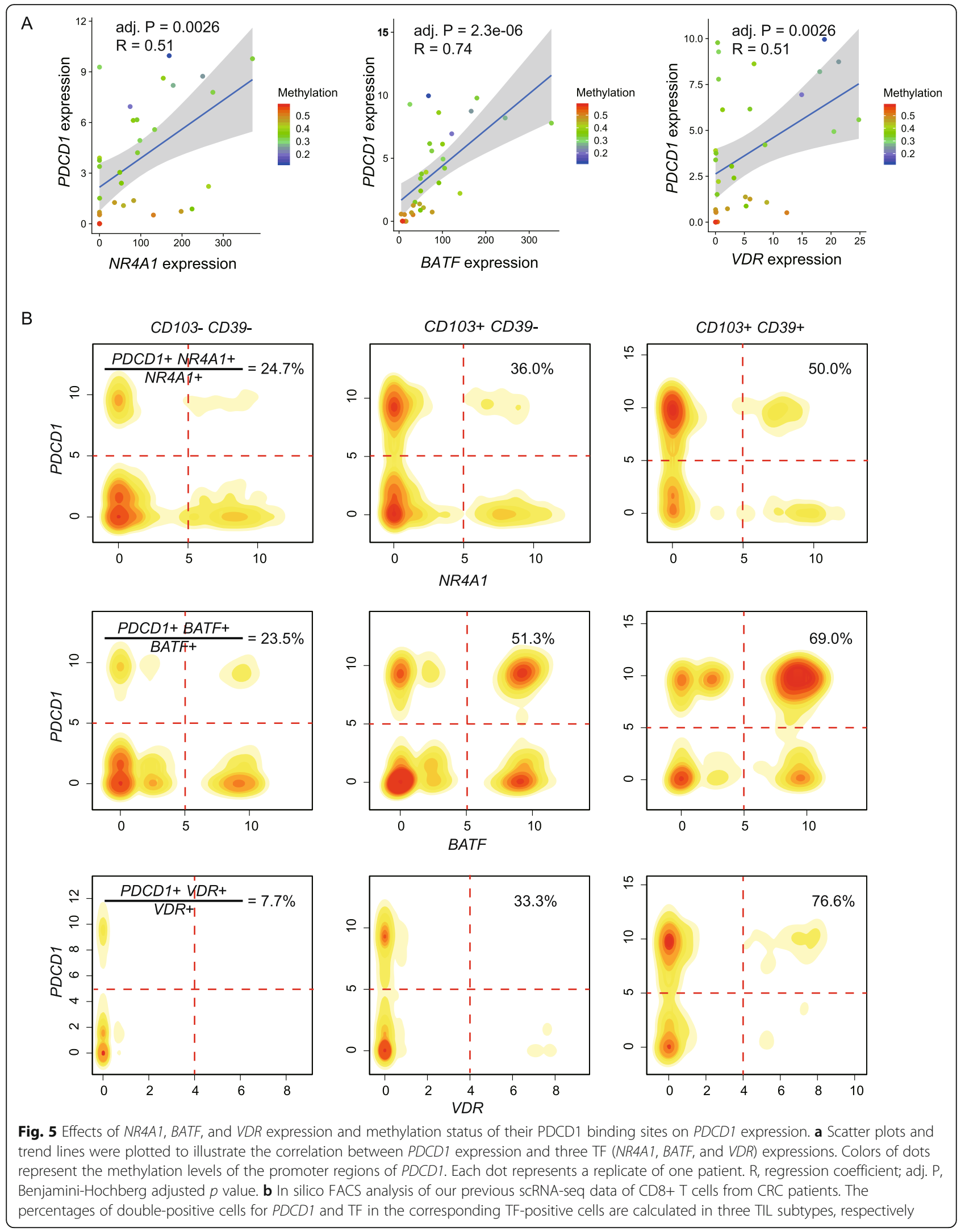


site, and suggested the contribution of these TFs in regulating $\mathrm{T}$ cell exhaustion.

Next, we analyzed the correlation of these three TFs with $T O X$, the novel $\mathrm{T}$ cell exhaustion marker. A strong positive correlation of TOX expression and $V D R$ expression was observed, which further supports a role of $V D R$ in regulating $\mathrm{T}$ cell exhaustion (Additional file 1: Figure S8). Future investigation should be considered that utilizes ChIP-seq to validate the exhaustion-associated targets of these TFs in tumor-reactive T cells.

\section{Discussion}

Intratumoral CD8+ $\mathrm{T}$ cells are classified as tumorreactive and bystanders based on their antigen specificities. Within CD8+ TILs, tumor-reactive $\mathrm{T}$ cells are enriched in $\mathrm{CD} 103+\mathrm{CD} 39+$ cells, while bystanders are abundant in CD39- cells [1, 3]. In eight patients, the proportions of tumor-reactive $\mathrm{T}$ cells ranged from 9.0 to $64.8 \%$, with an average of $29.3 \%$ (Additional file 1: Figure $\mathrm{S1B})$. A recent literature reported the intrinsic tumorrecognition potential of $\mathrm{T}$ cells in different human cancers by TCR profiling, and showed that tumor reactivity of TCRs was restricted to a minority of cells [2]. The relatively high proportion of tumor-reactive CD8+ T cells in our study suggested a certain number of bystander $\mathrm{T}$ cells were present. To further enrich tumorreactive $\mathrm{CD} 8+$ population in the future, new markers need to be identified in addition to CD39 and CD103. In addition, it is intriguing to find out the molecular mechanism determining the various proportions of tumorreactive $\mathrm{T}$ cells in different patients, which can have important clinical meanings. For instance, patients whose tumors had a higher percentage of tumor-reactive CD8 TILs at the time of surgery correlated with better overall survival [3]. Duhen et al. showed that the highest percentage of tumor-reactive CD8 TILs were found in melanoma and microsatellite instability (MSI) $)^{\text {high }}$ colon cancer, both tumors with high mutational burden [3]. Other molecular mechanism such as epigenetic regulation associated with proportions of tumor-reactive $\mathrm{T}$ cells remains to be explored in the future.

The exhausted state of tumor-reactive population impedes its therapeutic use. Reversing $\mathrm{T}$ cell exhaustion can reinvigorate immunity. However, a majority of patients lack durable response to immunotherapy such as immune checkpoint blockade, which is explained at least in part by the stable dysfunctional state of $\mathrm{T}$ cells shaped epigenetically [37, 38]. We added another layer of epigenetic regulation to intratumoral $\mathrm{T}$ cell exhaustion. Our in-depth methylation profiling identified the specific demethylation status of exhaustion markers, including PDCD1, HAVCR2, and LAYN. In addition, TF binding site analysis in HypoMRs for the enrichment of known TFs identified exhaustion-related TFs such as NR4A1,
$B A T F$, and EGR2. The current common epigenetic approaches for cancer treatment are the administration of demethylating agents such as azacitidine, which have a broad but undefined effect on the genome [39]. Advances in techniques for manipulating DNA methylation status in a targeted manner are anticipated to have significant clinical values [40-43]. Future therapeutic strategies of checkpoint blockade combined with epigenetic modifiers should be put into a brighter spotlight in the future.

We yielded putative biomarkers in mediating $\mathrm{T}$ cell exhaustion. Targeting these molecules might potentially augment $\mathrm{T}$ cell effector functions. Indeed, knockdown of $B A T F$ using shRNA-mediated gene-silencing enhanced $\mathrm{T}$ cell function [30]. NR4A1 deletion enhanced immunity against tumor and chronic virus [31]. Intriguingly, NR4A11-deficient CD8+ T cells have lower PDCD1 and $H A V C R 2$ expression [31]. In future study, roles of $V D R$ and RUNX1 in mediating $\mathrm{T}$ cells exhaustion need to be further defined and may provide new opportunities to reverse $\mathrm{CD} 8+\mathrm{T}$ cell exhaustion.

\section{Conclusion}

Methylation programming plays important roles in regulating gene expression. Herein, we showed that the transcriptomic features of tumor-reactive $\mathrm{T}$ cells were shaped by their distinct methylome profile. Intriguingly, tumor-reactive markers (CD39 and CD103) and exhaustion markers (PDCD1, LAYN, and HAVCR2) were specifically demethylated. Integrated transcriptome and methylome profiling identified possible key regulators in the tumor-reactive subtype, including exhaustion-related TFs such as NR4A1, BATF, and EGR2. Novel TFs RUNX1 and VDR identified here need further validation and may serve as potential therapeutic targets. Our understanding of transcriptome and methylome networks has important implications for the activation and expansion of tumor-reactive $\mathrm{T}$ cells, which will benefit future adoptive therapy.

\section{Methods}

\section{Human specimen collection}

This study was approved by the Research and Ethical Committee of Beijing Shijitan Hospital and complied with all relevant ethical regulations. Written informed consent was provided by all patients. Eight patients with CRC, including five women and three men, were enrolled and pathologically diagnosed with CRC at Beijing Shijitan Hospital. Fresh tumor and adjacent normal tissue samples (at least $2 \mathrm{~cm}$ from matched tumor tissues) were surgically resected from the above-described patients. None of them was treated with chemotherapy or radiation before tumor resection. The stages of these patients were classified according to the guidance of AJCC 
version 8 . The available clinical characteristics are summarized in Additional file 1: Figure S1A.

\section{Single cell collection}

Peripheral blood mononuclear cells (PBMCs) were isolated using HISTOPAQUE-1077 (Sigma-Aldrich) solution according to the manufacturer's instructions. Briefly, $3 \mathrm{~mL}$ of fresh peripheral blood was collected prior to surgery in EDTA anticoagulant tubes and subsequently layered onto HISTOPAQUE-1077. After centrifugation, lymphocyte cells remained at the plasmaHISTOPAQUE-1077 interface and were carefully transferred to a new tube and washed twice with $1 \times$ PBS (Invitrogen). These lymphocytes were re-suspended with FACS buffer (PBS supplemented with $1 \%$ fetal bovine serum (FBS, Invitrogen)).

Tumors and adjacent normal tissues were cut into approximately $1 \mathrm{~mm}^{3}$ pieces in the RPMI-1640 medium (Invitrogen), and mechanically dissociated and enzymatically digested with MACS Tumor Dissociation Kit (Miltenyi Biotec) for $30 \mathrm{~min}$ on a rotor at $37^{\circ} \mathrm{C}$, according to the manufacturer's instruction. The dissociated cells were subsequently passed through a $70-\mu \mathrm{m}$ cell-strainer (BD) and centrifuged at $400 \mathrm{~g}$ for $10 \mathrm{~min}$. The cell pellets were suspended in red blood cell lysis buffer (Solarbio) and incubated on ice for $2 \mathrm{~min}$ to lyse red blood cells. After washing twice with PBS (Invitrogen), the cell pellets were re-suspended in FACS buffer.

\section{Antibodies and flow cytometry}

The following fluorescent-labeled antibodies were used: BV711 anti-CD3 (BC96; 1:100-\#300450), APC antiCD8 (RPA-T8; 1:100—\#301048), BV421 anti-CD45RA (HI100; 1:100-\#47-0458-42), BV421 anti-CD45RO (HI100; 1:100-\#47-0458-42), BV421 anti-CCR7 (HI100; 1:100-\#47-0458-42), PE anti-CD39 (eBioA1; 1:100\#17-0399-42), and FITC anti-CD103 (B-Ly7 and BerACT8; 1:100-\#12-1038-42) (all from eBioscience). Cell surface staining was performed in FACS buffer. Stained cells were acquired on the FACS ArialI (all BD Biosciences) for cell sorting. Data were analyzed with FlowJo software (Treestar).

\section{Cell sorting, reverse transcription, amplification, and sequencing}

One thousand cells of different subtypes including naïve and $\mathrm{T}_{\mathrm{EM}} \mathrm{CD} 8+\mathrm{T}$ cells from PBMC, tumor-reactive $\mathrm{CD} 8+\mathrm{T}$ cells, and two clusters of tumor bystander $\mathrm{CD} 8+\mathrm{T}$ cells were enriched by gating 7AAD -CD3+CD8+CD45RO-CD45RA+CCR7+, 7AAD -CD3+CD8+CD45RO+CD45RA-CCR7-, 7AAD -CD3+CD8+CD103+CD39+, 7AAD $-\mathrm{CD} 3+\mathrm{CD} 8+\mathrm{CD} 103+\mathrm{CD} 39-, \quad$ and 7AAD $-\mathrm{CD} 3+\mathrm{CD} 8+\mathrm{CD} 103-\mathrm{CD} 39-$, respectively, and sorted into $0.2 \mathrm{~mL}$ tubes (Axygen) chilled to $4{ }^{\circ} \mathrm{C}$, prepared with lysis buffer with $1 \mu \mathrm{L} 10 \mathrm{mM}$ dNTP mix (Invitrogen), $1 \mu \mathrm{L} 10 \mu \mathrm{M}$ Oligo dT primer, $1.9 \mu \mathrm{L} 1 \%$ Triton X-100 (Sigma), and $0.1 \mu \mathrm{L} 40 \mathrm{U} \mu \mathrm{L}^{-1}$ RNase Inhibitor (Takara).

Transcriptome amplifications were performed according to Smart-Seq2 protocol [44] with modification of reagent amount and PCR cycle numbers. The amplified cDNA products were purified with Agencourt XP DNA beads (Beckman), and the concentration of each sample was quantified by Qubit HsDNA kits (Invitrogen). Libraries were constructed and amplified using the TruePrep DNA Library Prep Kit V2 for Illumina (Vazyme Biotech). The libraries were then purified with Agencourt XP DNA beads and analyzed by fragment analyzer for quality assessment. Purified libraries were analyzed by an Illumina Hiseq 4000 sequencer with 150-bp pairend reads.

\section{Whole-genome bisulfite-seq}

Whole-genome bisulfite-seq was performed according to a previously published protocol [45]. Briefly, 1000 cells were sorted into lysis buffer by FACS; DNA was released after proteinase treatment at $50^{\circ} \mathrm{C}$ and then subjected to bisulfite conversion. After bead-based purification, DNA was complemented with the biotinylated random primer Bio-P5-N9 (5'-biotin-CTACACGACGCTCTTCCG ATCTNNNNNNNNN-3') and 100 units of Klenow polymerase ( 3 ' to $5^{\prime}$ exo-, New England BioLabs). This random priming was repeated seven times in total. Second strands were synthesized using another random primer, P7-N9 (5'-AGACGTGTGCTCTTCCGATCTN NNNNNNNN-3'), and final libraries were generated after 7 to 9 cycles of PCR amplification with the Illumina universal PCR primer and Illumina indexed primer.

\section{RNA-seq analysis}

RNA-seq data were first processed to filter out lowquality reads with (1) "N" bases accounting for 3\% read length, or (2) bases with quality $<3$ account for $50 \%$ read length, or (3) containing adapter sequences. Then, kallisto [46] was used to quantify the abundances of transcripts. To summarize transcript-level abundance estimates for gene-level analysis, tximport [47] package from $\mathrm{R}$ bioconductor was used with parameter "countsFromAbundance $=$ lengthScaledTPM" to correct library size and average transcript length across samples. Differential gene expression analysis was performed by using DESeq2 [48] package from $\mathrm{R}$ Bioconductor. Only the genes with adjusted $p$ values less than 0.05 were considered to be differentially expressed. Normalized counts from DESeq2 were used to visualize the expression of genes and the downstream analysis. PCA analysis was performed with the $\mathrm{R}$ prcomp function on $\log 2$ (normalized counts +1$)$ expression values with specific gene 
subtypes, including highly variable genes across the five $\mathrm{T}$ cell populations (identified by $\mathrm{R}$ function FindVariableFeatures from Seurat [49] package) and significantly differential expression genes between CD103+CD39+ and CD103-CD39- samples. GSVA analysis was performed on $\log 2$ (normalized counts +1) expression values by using GSVA [50] $\mathrm{R}$ package. To test the functional enrichment of CD103+CD39+ TILs vs. CD103 -CD39- TILs, genes were ranked by fold-change difference, then using GSEA function from clusterProfiler [51] $R$ package to test enrichment of $T_{R M}$ and $T_{E X}$ signatures, collected from previously published paper [3].

Naive, cytotoxic, and exhausted scores were defined as the average expression of specific markers, with the expression level of each gene measured in log10-space. Seven markers for naïve $\mathrm{T}$ cells (CCR7, LEF1, SELL, TCF7, S1PR1, CD27, and CD28) were used for the naïve score. Eight markers for cytotoxic T cells (NKG7, CCL4, CST7, PRF1, GZMA, GZMB, IFNG, CCL3) and six markers for exhausted T cells (TIGIT, HAVCR2, CTLA4, PDCD1, LAG3, LAYN) were used to define the cytotoxic score and exhausted score. After delineating the naïve, cytotoxic, and exhausted scores of each $\mathrm{T}$ population, the Wilcoxon test was applied to compare the difference between each group, with $p$ value $<0.05$ considered significant.

\section{Bisulfite-seq analysis}

Sequencing reads were trimmed of 9 bases for random primer sequences and removed the low-quality and adapter contaminated reads using trim galore (http:// www.bioinformatics.babraham.ac.uk/projects/trim_galore). The cleaned reads were then mapped to the computationally bisulfite-converted human reference genome (GRCh38) by using Bismark [52] with pairedend mode (parameter settings: "bismark --pbat -N 1 - $\mathrm{L}$ 32 "). Then, the unmapped reads after paired-end mapping were re-aligned to the same reference in single-end mode. Potential PCR duplicates were removed using $d u$ plicate-remover in MethPipe [53]. The lambda genome was added into human reference genome as extra chromosome to estimate the bisulfite conversion rate. We used bsrate from MethPipe to estimate bisulfite conversion rate, and samples with $>99 \%$ (or almost 99\%) bisulfite conversion rates were retained for the DNA methylation analysis. Most post-alignment analysis was performed by functions from MethPipe [53] Package.

Methylation levels for each symmetric CpG site were calculated by the methcounts and symmetric-cpgs commands in MethPipe. Average CpG methylation levels of about $5 \mathrm{~kb}$ tils for all chromosomes in human genome were calculated with MethPipe roimethstat command for each $\mathrm{T}$ cell population. Only high-confidence genomic regions with at least $40 \mathrm{CpG}$ observations from reads in the genomic tile were used for the PCA analysis ( $\mathrm{R}$ prcomp function) to compare the overall methylation level in genome wide of the five $\mathrm{T}$ cell populations.

Specific hypomethylated regions (HypoMRs) for each $\mathrm{T}$ cell population were calculated in a "one vs. rest" fashion by using radmeth regression, radmeth adjust, and radmeth merge commands in MethPipe. Then, we overlapped these HypoMRs with promoter regions (defined as $-2.5 \mathrm{~kb}$ and $1 \mathrm{~kb}$ from transcription start site (RefSeq gene model downloaded from UCSC Table browser [54])) to identify genes affected by HypoMRs in each $\mathrm{T}$ cell population. The average methylation level of all HypoMRs in each $\mathrm{T}$ cell population was calculated by roimethstat command. To visualize the methylation pattern of given genes in different populations, lollipop plot from CGmapTools [55] was used. The Wilcoxon test was applied to compare the methylation levels of HypoMR between each group, with $p$ value $<0.05$ considered significant.

\section{Correlation of promoter methylation and RNA expression of immune-related genes}

Here, we focused on three immune signature gene sets for naïve (CCR7, LEF1, SELL, TCF7, S1PR1, CD27, and CD28), cytotoxic (NKG7, CCL4, CST7, PRF1, GZMA, GZMB, IFNG, and CCL3) and exhausted (TIGIT, HAVCR2, CTLA4, PDCD1, LAG3, and LAYN) T cells. First, the average methylation levels of those gene promoter regions were calculated by using roimethstat command in each sample. RNA expression level of those genes was calculated by using the normalized counts from DESeq2. Spearman's correlation was used to calculate the relationship of RNA expression level and promoter methylation level. $p$ values were adjusted by the Benjamini-Hochberg method.

\section{Inferred regulation network construction}

First, the enriched transcription factor motifs within HypoMRs were performed by HOMER [56] to identify possible key transcription factors in each $\mathrm{T}$ cell population. Furthermore, to investigate the possible target genes regulated by transcription factors in tumorreactive $\mathrm{T}$ cells, transcription factor binding motifs were scanned through the promoter regions in the whole human genome (using scanMotifGenomeWide.pl in homer) to identify all possible target sites genome wide, and then, all the predicted target sites were intersected with the specific hypomethylated regions in tumor-reactive $\mathrm{T}$ cell population to construct the possible regulation network in each $\mathrm{T}$ cell population.

\section{In silico FACS for scRNA-seq data}

scRNA-Seq was downloaded from GSE108989 [19]. A total of $1646 \mathrm{CD} 8+\mathrm{T}$ cells from tumor site were 
retained for our analysis, with the expression level of each gene measured by $\log 2(\mathrm{TPM}+1)$. Using cutoff 4 for the expression of gene CD103 and CD19, 25.9\% cells were classified as $\mathrm{CD} 103+\mathrm{CD} 39+, \quad 41.0 \%$ were CD103+CD39-, and 29.9\% were CD103-CD39-. Then, we used the scRNA-Seq data to investigate the coexpression of PDCD1 and three TFs (NR4A1, BATF, and $V D R)$. The percentages of double-positive cells for $P D C D 1$ and TF in the corresponding TF-positive cells are calculated in the three TIL subsets, respectively.

\section{Supplementary information}

Supplementary information accompanies this paper at https://doi.org/10. 1186/s13059-019-1921-y.

Additional file 1. Contains supplemental figures and table outlining the analysis of transcriptome and methylome data from 5 CD8 T cell subtypes of 8 patients.

Additional file 2. Review history.

\section{Acknowledgements}

The authors would like to thank Fei Wang, Li Yang, and Xuefang Zhang for assistance with FACS. We thank Dr. Fuchou Tang for bisulfite sequencing technical assistance. We thank the members of the BIOPIC high-throughput sequencing facility and the Computing Platform of the Centre for Life Science.

\section{Peer review information}

Yixin Yao was the primary editor on this article and managed its editorial process and peer review in collaboration with the rest of the editorial team.

\section{Review history}

The review history is available as Additional file 2

\section{Authors' contributions}

$Z Z, R Y, S C, X R, J P$, and $A H$ designed the experiments. RY, RG, KY, BK, LW, QZ, $\mathrm{QF}$, and $\mathrm{CL}$ performed the experiments. SC, RY, $X R$, and $L Z$ analyzed the sequencing data. NL, RY, RG, KY, and LW collected the clinical samples. RY, SC, and ZZ wrote the manuscript with all authors contributing to writing and providing feedback. All authors read and approved the final manuscript.

\section{Funding}

This work was supported by grants from the Beijing Advanced Innovation Center for Genomics at Peking University and the National Natural Science Foundation of China (81573022, 31530036, and 91742203).

\section{Availability of data and materials}

All the sequencing data were deposited into Genome Sequence Archive database under accession number HRA000059 [57] (https://bigd.big.ac.cn/ gsa-human/browse/HRA000059) and GSE141878 (https://www.ncbi.nlm.nih. gov/geo/query/acc.cgi?acc=GSE141878) [58]. ScRNA-Seq data was downloaded from GSE108989 [19] for in silico FACS.

\section{Ethics approval and consent to participate}

This study was approved by the Research and Ethical Committee of Beijing Shijitan Hospital and complied with all relevant ethical regulations. Written informed consent was provided by all patients. All experimental methods abided by the Helsinki Declaration.

\section{Consent for publication}

Not applicable.

\section{Competing interests}

The authors declare that they have no competing interests.

\section{Author details}

'BIOPIC, Beijing Advanced Innovation Center for Genomics, and School of Life Sciences, Peking University, Beijing, China. ${ }^{2}$ Department of Surgery, Beijing Shijitan Hospital, Capital Medical University, Beijing, China.

${ }^{3}$ Department of Surgery, Peking University Ninth School of Clinical Medicine, Beijing, China. ${ }^{4}$ Peking-Tsinghua Center for Life Sciences, Academy for Advanced Interdisciplinary Studies, Peking University, Beijing, China. ${ }^{5}$ Institute of Molecular Medicine, Peking-Tsinghua Center for Life Sciences, Beijing Key Laboratory of Cardiometabolic Molecular Medicine, Peking University, Beijing, China.

Received: 23 July 2019 Accepted: 12 December 2019

Published online: 31 December 2019

\section{References}

1. Simoni Y, Becht E, Fehlings M, Loh CY, Koo SL, Teng KWW, et al. Bystander CD8(+) T cells are abundant and phenotypically distinct in human tumour infiltrates. Nature. 2018;557(7706):575-9.

2. Scheper W, Kelderman S, Fanchi LF, Linnemann C, Bendle G, MAJ d R, et al. Low and variable tumor reactivity of the intratumoral TCR repertoire in human cancers. Nat Med. 2018;25(1):89-94.

3. Duhen T, Duhen R, Montler R, Moses J, Moudgil T, de Miranda NF, et al. Coexpression of CD39 and CD103 identifies tumor-reactive CD8 T cells in human solid tumors. Nat Commun. 2018;9(1):2724

4. Li H, van der Leun AM, Yofe I, Lubling Y, Gelbard-Solodkin D, van Akkooi ACJ, et al. Dysfunctional CD8 T cells form a proliferative, dynamically regulated compartment within human melanoma. Cell. 2018;176(4):775-89

5. Willinger T, Freeman T, Hasegawa H, McMichael AJ, Callan MF. Molecular signatures distinguish human central memory from effector memory CD8 T cell subsets. J Immunol. 2005;175(9):5895-903.

6. Bonasio R, Tu S, Reinberg D. Molecular signals of epigenetic states. Science. 2010;330(6004):612-6.

7. Jones PA. Functions of DNA methylation: islands, start sites, gene bodies and beyond. Nat Rev Genet. 2012;13(7):484-92.

8. Bintu L, Yong J, Antebi YE, McCue K, Kazuki Y, Uno N, et al. Dynamics of epigenetic regulation at the single-cell level. Science. 2016;351(6274):720-4.

9. Razin A, Cedar H. DNA methylation and gene expression. Microbiol Rev. 1991;55(3):451-8.

10. Kwon NH, Kim JS, Lee JY, Oh MJ, Choi DC. DNA methylation and the expression of IL-4 and IFN-gamma promoter genes in patients with bronchial asthma. J Clin Immunol. 2008;28(2):139-46.

11. Melvin AJ, McGurn ME, Bort SJ, Gibson C, Lewis DB. Hypomethylation of the interferon-gamma gene correlates with its expression by primary T-lineage cells. Eur J Immunol. 1995;25(2):426-30.

12. Komori HK, Hart T, LaMere SA, Chew PV, Salomon DR. Defining CD4 T cell memory by the epigenetic landscape of CPG DNA methylation. J Immunol. 2015;194(4):1565-79.

13. Intlekofer AM, Takemoto N, Wherry EJ, Longworth SA, Northrup JT, Palanivel VR, et al. Effector and memory CD8+ T cell fate coupled by T-bet and eomesodermin. Nat Immunol. 2005;6(12):1236-44.

14. Gerlach C, Moseman EA, Loughhead SM, Alvarez D, Zwijnenburg AJ, Waanders $L$, et al. The chemokine receptor CX3CR1 defines three antigenexperienced CD8 T cell subsets with distinct roles in immune surveillance and homeostasis. Immunity. 2016;45(6):1270-84.

15. Khan O, Giles JR, McDonald S, Manne S, Ngiow SF, Patel KP, et al. TOX transcriptionally and epigenetically programs CD8(+) T cell exhaustion. Nature. 2019:571(7764):211-18.

16. Alfei F, Kanev K, Hofmann M, Wu M, Ghoneim HE, Roelli P, et al. TOX reinforces the phenotype and longevity of exhausted T cells in chronic viral infection. Nature. 2019;571(7764):265-69.

17. Scott AC, Dundar F, Zumbo P, Chandran SS, Klebanoff CA, Shakiba M, et al. TOX is a critical regulator of tumour-specific T cell differentiation. Nature. 2019;571(7764):270-74.

18. Yao C, Sun HW, Lacey NE, Ji Y, Moseman EA, Shih HY, et al. Single-cell RNAseq reveals TOX as a key regulator of CD8(+) T cell persistence in chronic infection. Nat Immunol. 2019;20(7):890-901.

19. Zhang L, Yu X, Zheng L, Zhang Y, Li Y, Fang Q, et al. Lineage tracking reveals dynamic relationships of T cells in colorectal cancer. Nature. 2018; 564(7735):268-72. 
20. Zheng C, Zheng L, Yoo JK, Guo H, Zhang Y, Guo X, et al. Landscape of infiltrating $T$ cells in liver cancer revealed by single-cell sequencing. Cell. 2017;169(7):1342-56 e16

21. Guo X, Zhang Y, Zheng L, Zheng C, Song J, Zhang Q, et al. Global characterization of $T$ cells in non-small-cell lung cancer by single-cell sequencing. Nat Med. 2018;24(7):978-85.

22. Ejrnaes M, Filippi CM, Martinic MM, Ling EM, Togher LM, Crotty S, et al. Resolution of a chronic viral infection after interleukin-10 receptor blockade. J Exp Med. 2006;203(11):2461-72.

23. Castro F, Cardoso AP, Goncalves RM, Serre K, Oliveira MJ. Interferon-gamma at the crossroads of tumor immune surveillance or evasion. Front Immunol. 2018;9:847.

24. Perng YC, Lenschow DJ. ISG15 in antiviral immunity and beyond. Nat Rev Microbiol. 2018;16(7):423-39.

25. Kratchmarov R, Magun AM, Reiner SL. TCF1 expression marks self-renewing human CD8(+) T cells. Blood Adv. 2018;2(14):1685-90.

26. Willinger T, Freeman T, Herbert M, Hasegawa H, McMichael AJ, Callan MF. Human naive CD8 T cells down-regulate expression of the WNT pathway transcription factors lymphoid enhancer binding factor 1 and transcription factor 7 ( $T$ cell factor-1) following antigen encounter in vitro and in vivo. J Immunol. 2006;176(3):1439-46.

27. Pearce EL, Mullen AC, Martins GA, Krawczyk CM, Hutchins AS, Zediak VP, et al. Control of effector CD8+ T cell function by the transcription factor Eomesodermin. Science. 2003;302(5647):1041-3.

28. Godec J, Cowley GS, Barnitz RA, Alkan O, Root DE, Sharpe AH, et al. Inducible RNAi in vivo reveals that the transcription factor BATF is required to initiate but not maintain CD8+ T-cell effector differentiation. Proc Natl Acad Sci U S A. 2015;112(2):512-7.

29. Kurachi M, Barnitz RA, Yosef N, Odorizzi PM, Dilorio MA, Lemieux ME, et al. The transcription factor BATF operates as an essential differentiation checkpoint in early effector CD8+ T cells. Nat Immunol. 2014;15(4):373-83.

30. Quigley M, Pereyra F, Nilsson B, Porichis F, Fonseca C, Eichbaum Q, et al. Transcriptional analysis of HIV-specific CD8+ T cells shows that PD-1 inhibits T cell function by upregulating BATF. Nat Med. 2010;16(10):1147-51.

31. Liu X, Wang Y, Lu H, Li J, Yan X, Xiao M, et al. Genome-wide analysis identifies NR4A1 as a key mediator of T cell dysfunction. Nature. 2019; 567(7749):525-9.

32. Williams JB, Horton BL, Zheng Y, Duan Y, Powell JD, Gajewski TF. The EGR2 targets $L A G-3$ and $4-1 B B$ describe and regulate dysfunctional antigenspecific CD8+ T cells in the tumor microenvironment. J Exp Med. 2017. 214(2):381-400.

33. Woolf E, Xiao C, Fainaru O, Lotem J, Rosen D, Negreanu V, et al. Runx3 and Runx1 are required for CD8 T cell development during thymopoiesis. Proc Natl Acad Sci U S A. 2003;100(13):7731-6.

34. Kongsbak M, Levring TB, Geisler C, von Essen MR. The vitamin d receptor and T cell function. Front Immunol. 2013:4:148.

35. Suzuki T, Shimizu Y, Furuhata E, Maeda S, Kishima M, Nishimura H, et al. RUNX1 regulates site specificity of DNA demethylation by recruitment of DNA demethylation machineries in hematopoietic cells. Blood Adv. 2017; 1(20):1699-711.

36. Ronchetti S, Nocentini G, Riccardi C, Pandolfi PP. Role of GITR in activation response of T lymphocytes. Blood. 2002;100(1):350-2.

37. Philip M, Fairchild L, Sun L, Horste EL, Camara S, Shakiba M, et al. Chromatin states define tumour-specific T cell dysfunction and reprogramming. Nature. 2017:545(7655):452-6.

38. Pauken KE, Sammons MA, Odorizzi PM, Manne S, Godec J, Khan O, et al. Epigenetic stability of exhausted T cells limits durability of reinvigoration by PD-1 blockade. Science. 2016;354(6316):1160-5.

39. Emran AA, Chatterjee A, Rodger EJ, Tiffen JC, Gallagher SJ, Eccles MR, et al. Targeting DNA methylation and EZH2 activity to overcome melanoma resistance to immunotherapy. Trends Immunol. 2019;40(4):328-44.

40. Xu X, Tao Y, Gao X, Zhang L, Li X, Zou W, et al. A CRISPR-based approach for targeted DNA demethylation. Cell Discov. 2016;2:16009.

41. McDonald JI, Celik H, Rois LE, Fishberger G, Fowler T, Rees R, et al. Reprogrammable CRISPR/Cas9-based system for inducing site-specific DNA methylation. Biol Open. 2016;5(6):866-74.

42. Morita S, Noguchi H, Horii T, Nakabayashi K, Kimura M, Okamura K, et al. Targeted DNA demethylation in vivo using dCas9-peptide repeat and scFvTET1 catalytic domain fusions. Nat Biotechnol. 2016;34(10):1060-5.
43. Huang $Y H$, Su J, Lei Y, Brunetti L, Gundry MC, Zhang X, et al. DNA epigenome editing using CRISPR-Cas SunTag-directed DNMT3A. Genome Biol. 2017;18(1):176.

44. Picelli S, Faridani OR, Bjorklund AK, Winberg G, Sagasser S, Sandberg R. Fulllength RNA-seq from single cells using Smart-seq2. Nat Protoc. 2014;9(1): 171-81.

45. Smallwood SA, Lee HJ, Angermueller C, Krueger F, Saadeh H, Peat J, et al. Single-cell genome-wide bisulfite sequencing for assessing epigenetic heterogeneity. Nat Methods. 2014;11(8):817-20.

46. Bray NL, Pimentel H, Melsted P, Pachter L. Near-optimal probabilistic RNAseq quantification. Nat Biotechnol. 2016;34(5):525-7.

47. Soneson C, Love MI, Robinson MD. Differential analyses for RNA-seq: transcript-level estimates improve gene-level inferences. F1000Res. 2015;4: 1521

48. Love Ml, Huber W, Anders S. Moderated estimation of fold change and dispersion for RNA-seq data with DESeq2. Genome Biol. 2014;15(12):550.

49. Butler A, Hoffman P, Smibert P, Papalexi E, Satija R. Integrating single-cell transcriptomic data across different conditions, technologies, and species. Nat Biotechnol. 2018;36(5):411-20.

50. Hanzelmann S, Castelo R, Guinney J. GSVA: gene set variation analysis for microarray and RNA-seq data. BMC Bioinformatics. 2013;14:7.

51. Yu G, Wang LG, Han Y, He QY. clusterProfiler: an R package for comparing biological themes among gene clusters. OMICS. 2012;16(5):284-7.

52. Krueger F, Andrews SR. Bismark: a flexible aligner and methylation caller for Bisulfite-Seq applications. Bioinformatics. 2011;27(11):1571-2.

53. Song $Q$, Decato $B$, Hong EE, Zhou M, Fang F, Qu J, et al. A reference methylome database and analysis pipeline to facilitate integrative and comparative epigenomics. PLoS One. 2013;8(12):e81148.

54. Haeussler M, Zweig AS, Tyner C, Speir ML, Rosenbloom KR, Raney BJ, et al. The UCSC genome browser database: 2019 update. Nucleic Acids Res. 2019; 47(D1):D853-D8

55. Guo W, Zhu P, Pellegrini M, Zhang MQ, Wang X, Ni Z. CGmapTools improves the precision of heterozygous SNV calls and supports allelespecific methylation detection and visualization in bisulfite-sequencing data. Bioinformatics. 2018:34(3):381-7.

56. Heinz S, Benner C, Spann N, Bertolino E, Lin YC, Laslo P, et al. Simple combinations of lineage-determining transcription factors prime cisregulatory elements required for macrophage and B cell identities. Mol Cell. 2010;38(4):576-89.

57. Yang R, Cheng SJ, Luo N, Gao RR, Yu KZ, Kang BX, Wang L, Zhang QM, Fang Q, Zhang L, Li C, He AB, Hu XD, Peng JR, Ren XW, Zhang ZM. Transcriptomic and epigenetic features of tumor-reactive CD8+ T cells in colorectal cancer patients. Datasets. Genome Sequence Archive. 2019; https://bigd.big.ac.cn/ gsa-human/browse/HRA000059. Accessed 03 Dec 2019.

58. Yang R, Cheng SJ, Luo N, Gao RR, Yu KZ, Kang BX, Wang L, Zhang QM, Fang Q, Zhang L, Li C, He AB, Hu XD, Peng JR, Ren XW, Zhang ZM. Transcriptomic and epigenetic features of tumor-reactive CD8+ T cells in colorectal cancer patients. Datasets. Gene Expression Omnibus. 2019 https://www.ncbi.n/m.nih.gov/geo/query/acc.cgi?acc=GSE141878. Accessed 12 Dec 2019

\section{Publisher's Note}

Springer Nature remains neutral with regard to jurisdictional claims in published maps and institutional affiliations.

Ready to submit your research? Choose BMC and benefit from:

- fast, convenient online submission

- thorough peer review by experienced researchers in your field

- rapid publication on acceptance

- support for research data, including large and complex data types

- gold Open Access which fosters wider collaboration and increased citations

- maximum visibility for your research: over $100 \mathrm{M}$ website views per year

At $\mathrm{BMC}$, research is always in progress.

Learn more biomedcentral.com/submissions 